\title{
Atividades didáticas de Física mediadas por hipermídia: potencialidades para o desenvolvimento da flexibilidade cognitiva
}

Didactic activities of Physics mediated by hypermedia: potentialities for the development of the cognitive flexibility

\author{
Muryel Pyetro Vidmar*1], Inés Prieto Schmidt Sauerwein ${ }^{10}$ \\ ${ }^{1}$ Universidade Federal de Santa Maria, Santa Maria, RS, Brasil
}

Recebido em 28 de janeiro de 2020. Revisado em 04 de agosto de 2020. Aceito em 12 de agosto de 2020.

\begin{abstract}
Apresentamos neste artigo um conjunto de atividades didáticas (AD) de Física, mediadas por recursos educacionais hipermídia, que aborda a energia elétrica nas residências, relacionando conteúdos de Física referentes à Eletrodinâmica. Para isso, consideramos a importância da recontextualização dos conhecimentos científicos explicitada por diversos autores e pelos documentos oficiais voltados ao ensino médio de Física - que possibilita aos estudantes lidar com as situações que vivenciam ou venham a vivenciar, muitas delas novas e inéditas. Nesse sentido, as AD foram estruturadas tendo como base teórico-metodológica a Teoria da Flexibilidade Cognitiva (TFC), que enfatiza o desenvolvimento da flexibilidade cognitiva (FC) como essencial na construção, organização e reestruturação do conhecimento face a novas situações e contextos de utilização. Ao longo do texto, descrevemos as etapas envolvidas no planejamento das atividades, bem como analisamos os avanços e obstáculos na estruturação das mesmas em consonância com os pressupostos da TFC. As potencialidades das AD também são discutidas, com ênfase na contribuição destas para o desenvolvimento da FC no âmbito do ensino médio.
\end{abstract}

Palavras-chave: Atividade didática de Física, hipermídia, flexibilidade cognitiva, ensino médio, eletrodinâmica.

In this paper we present a set of didactic activities (DA) of Physics, mediated by hypermedia educational resources, that approach electrical energy in homes, relating Physics contents from Electrodynamics. For this, we consider the importance to recontextualize scientific knowledge - explained by several authors and by the official documents aimed at the high school of Physics - that allows students deal with the situations they experience or may experience, many of them new and original. In this context, the DA were structured with the theoretical-methodological basis of the Cognitive Flexibility Theory (CFT), that emphasizes the development of cognitive flexibility $(\mathrm{CF})$ as essential in the construction, organization and restructuring of knowledge in the face of new situations and contexts of use. Throughout the text, we describe the steps related in the planning of activities, as well as analyzing the advances and obstacles in structuring them in accordance with the assumptions of the TFC. The potentialities of DA are also discussed, with an emphasis on their contribution to the development of $\mathrm{CF}$ within the scope of high school.

Keywords: Didactic activity of Physics, hypermedia, cognitive flexibility, high school, electrodynamics.

\section{Introdução}

Políticas públicas educacionais voltadas ao ensino médio de Física - como os PCN+ [1], as Orientações Curriculares para o Ensino Médio [2] e as Diretrizes Curriculares Nacionais da Educação Básica [3] - explicitam a ideia de que a formação geral que a escola precisa proporcionar aos seus estudantes tem como objetivo principal ampliar a compreensão que eles têm do mundo em que vivem.

Este objetivo mais amplo requer, sobretudo, que os estudantes se apropriem de conhecimentos científicos e desenvolvam competências para lidar com as situações que vivenciam ou que venham a vivenciar, muitas delas novas e inéditas $[1,3]$.

*Endereço de correspondência: muryel.vidmar@ufsm.br
Em situações complexas e/ou inéditas, é preciso que o estudante seja capaz de recontextualizar os conhecimentos construídos para compreendê-las [4]. A noção de competências pretende que o estudante "mobilize seus conhecimentos em contextos distintos daquele em que aprendeu, para poder se relacionar com o mundo". [2, p. 48].

Nesta linha, Pozo e Crespo [5] abordam a velocidade de mudança dos conhecimentos e das demandas formativas na sociedade atual, enfatizando a importância de que os sujeitos sejam aprendizes flexíveis que possam dispor de procedimentos e capacidades de aprendizagem que os possibilite adaptar-se às referidas demandas. Os autores enfatizam que a meta da educação precisa ser "[...] tornar mais transferível e generalizável o conhecimento. A 
aprendizagem escolar deve ser situada em sua origem, em seu ponto de partida, mas suas metas devem ser mais gerais, devem facilitar a transferência do conhecimento de um contexto para outro" [5, p. 129, grifo do autor].

A Teoria da Flexibilidade Cognitiva (TFC), proposta no final da década de 80 por Rand Spiro e colaboradores, vai ao encontro das referidas preocupações. Ela enfatiza o desenvolvimento da flexibilidade cognitiva (FC) como essencial na construção, organização e reestruturação do conhecimento face a novas situações e contextos de utilização [6]. Os pressupostos dessa teoria foram desenvolvidos a partir da constatação de que os estudantes tinham dificuldade em recontextualizar conhecimentos para novas situações $[7,8]$.

Em relação ao ensino de Ciências, Pozo e Crespo [5] apontam que é muito comum que algo aprendido pelos estudantes se dilua ou se torne difuso quando se trata de aplicar esse conhecimento a um problema ou situação nova. Os autores também citam o caso específico dos problemas quantitativos, nos quais se observa fraca generalização dos procedimentos construídos para outros contextos. Isso porque, assim que é alterado o formato ou o conteúdo conceitual do problema, os estudantes apresentam dificuldades em aplicar o que foi aprendido a essa nova situação [5].

Em consonância, Echeverría e Pozo [9] destacam que a reestruturação dos conhecimentos construídos em um novo contexto "[...] constitui o problema de aprendizagem mais difícil de superar, tanto para as teorias da aprendizagem como para a própria prática didática e educacional". [9, p. 41]. Os autores abordam que essa reestruturação se torna difícil de um assunto para outro e de uma área para outra, mas especialmente quando se trata de um conhecimento construído em aula para um contexto mais cotidiano. Essa dificuldade ocorre principalmente devido à diferença entre os contextos nos quais o estudante aprende inicialmente e os novos contextos para os quais precisa fazer a recontextualização [9].

Neste contexto, fundamentados pelos pressupostos da TFC, e considerando a dificuldade na recontextualização dos conhecimentos científicos [5, 9] e a importância da FC, apresentamos, neste trabalho, um conjunto de atividades didáticas $(\mathrm{AD})$ de Física mediadas por hipermídia, bem como discutimos as potencialidades das mesmas para consubstanciar o desenvolvimento da FC no âmbito do ensino médio.

\section{Conceitos Guia no Planejamento das AD de Física}

\subsection{Hipermídia}

O conceito de hipermídia é complexo e está diretamente associado a outros dois conceitos: hipertexto e multimídia [10-14].

Por hipertexto entendemos um sistema computacional que representa nós de informações textuais, organizadas de modo não linear, através de ligações conceituais [10,
11, 14, 15]. Nessa linha, Da Silva [13] aborda o hipertexto como um texto digital composto por blocos de conteúdos interligados por links - que geralmente são outros textos -, permitindo que o estudante faça uma leitura na ordem que desejar e proporcionando conexões entre os conceitos que possam levar à formação de significados relevantes para ele $[11,15]$.

Já multimídia compreende a conjugação em uma mesma tecnologia de diversas mídias - tais como textos, áudios, imagens, vídeos, animações e simulações - para representar uma informação [11, 15], mas sem ou com poucas possibilidades de alteração na ordem da apresentação das informações; ou seja, com pouca interatividade [10, 13, 14]. Essa diversidade de mídias "[...] permite que a informação seja apresentada segundo múltiplas representações, reforçando as ideias contidas nos textos e ampliando as possibilidades para associações pertinentes dos conceitos na estrutura cognitiva do aluno" [11, p. 475-476].

Nesse sentido, Rezende e Cola [10] afirmam que a hipermídia consiste na interseção entre multimídia e hipertexto. Isso porque se trata de sistemas computacionais que ligam informações de forma não linear, como no hipertexto, e que utilizam diversos meios para representar a informação, como os materiais multimídia.

Nessa linha, Coutinho [16] se refere à hipermídia como uma organização não linear de acesso à informação, tendo como suporte o computador em rede. Essa informação, de acordo com a autora, pode integrar diversas mídias simultaneamente, como áudios, imagens, gráficos e vídeos. Salgado [17] aborda o fato de que funcionar em rede traz à hipermídia a vantagem de poder ser amplamente acessada.

Da Silva [13] cita que a hipermídia educacional possui características diferentes daquelas planejadas para outros fins. Nesse caso, há uma intencionalidade explícita de conduzir o estudante a uma navegação por um documento que é voltado para um determinado domínio de conhecimento, em que o ponto central na hipermídia é o conteúdo.

Assim, é essencial que o professor, considerando os objetivos de seu planejamento didático, forneça possíveis caminhos para direcionar o estudante na interatividade com o material, atentando para que ele não se perca nem se distancie do conteúdo específico que está sendo abordado [13].

\subsection{Flexibilidade cognitiva}

\subsubsection{Flexibilidade cognitiva (FC) no âmbito da Teoria da Flexibilidade Cognitiva (TFC)}

Quando diante de situações complexas e/ou novas situações, é necessário que o sujeito seja capaz de reestruturar conhecimentos e desenvolver novo repertório comportamental para compreendê-las. É nesse contexto que surge a importância da FC.

$\mathrm{O}$ conceito de $\mathrm{FC}$ é estudado por vários autores, que atribuem a ele diversas interpretações e significados [18]. Optamos nesse trabalho pela perspectiva contida no âm- 
bito da TFC, visto que ela: aborda o conceito de FC com ênfase nos processos de ensino-aprendizagem; discute aspectos necessários para o desenvolvimento da FC; e apresenta estratégias para consubstanciar esses aspectos e, com isso, desenvolver a FC.

A TFC consiste em uma teoria de ensino-aprendizagem, representação do conhecimento e recontextualização do conhecimento $[6,19,20]$, desenvolvida por Rand Spiro e colaboradores no final da década de 80 [21].

O foco de aplicação dessa teoria está na construção de conhecimento de nível avançado em domínios pouco estruturados [6, 21-24], tendo sido desenvolvida como uma resposta às dificuldades apresentadas no referido contexto [8, 19, 25-27].

O objetivo principal da TFC é promover o desenvolvimento da FC [24, 25, 28], que consiste na "capacidade que o sujeito desenvolve de, perante uma situação nova, reestruturar o conhecimento para a solucionar" [29, p. 1]. Nesse contexto, a recontextualização de conhecimentos está relacionada com a aplicação do que foi aprendido a novas situações.

Uma situação nova de aplicação de conhecimento envolve a combinação de diversos conceitos [20], podendo estar associada, por exemplo, a um problema a ser resolvido $[6,23]$. O aspecto central na identificação dessa situação nova é que nela precisa haver características que a diferem da situação de aprendizagem [21].

A base para o desenvolvimento da FC consiste na capacidade de reorganização do conhecimento necessário para resolver uma situação nova [24]. Para se obter essa reestruturação, é preciso que o conhecimento seja construído de uma forma particular [21, 23].

A TFC se estrutura a partir do referido objetivo principal e, nesse sentido, visa proporcionar ao sujeito a construção de esquemas flexíveis ao longo do processo de construção do conhecimento. Uma das mais importantes recomendações para essa construção flexível é a utilização de múltiplas representações do conhecimento [6], que favoreçam a reestruturação desse para novas situações $[10,30]$.

As múltiplas representações do conhecimento se referem a dois aspectos principais: i) múltiplas formas de organização do conhecimento, por meio de casos, mini casos e temas de análise conceitual interligados [19, 31, 32]; e ii) múltiplas mídias - textos, imagens, gráficos, animações, vídeos - para apresentação do conhecimento (caso, mini caso e tema) [20].

A TFC aborda outros aspectos necessários para o desenvolvimento da FC [10, 20-23, 30, 31, 33]: i) concepção do conhecimento conceitual como conhecimento em utilização, de modo que o significado de um conceito está vinculado ao seu contexto de aplicação; ii) enfoque no caso como unidade de análise dos domínios de conhecimento; iii) análise de muitos casos diferentes; iv) desconstrução de casos e mini casos através de diferentes temas; v) estabelecimento de ligações entre mini casos de diferentes casos; vi) construção flexível de conhecimento; vii) promoção de diversas ligações entre conceitos e casos.
É importante destacar a dificuldade de implementação da TFC, apontada por seus autores, tendo em vista a diversidade de aspectos envolvidos que procuramos mencionar [27].

De modo a contribuir para consubstanciar o desenvolvimento da FC, a TFC apresenta algumas estratégias, as quais serão discutidas a seguir.

\subsubsection{Estratégias de ensino para o desenvolvimento da FC}

A TFC apresenta estratégias de ensino para o desenvolvimento da FC, estruturadas a partir dos seguintes elementos: caso, mini caso, tema de análise conceitual, comentário temático e travessia temática [6].

Buscando dar resposta ao desenvolvimento do domínio da complexidade e da capacidade de reestruturar o conhecimento de maneira flexível para situações novas, a TFC utiliza uma abordagem centrada no estudo de casos [20, 23, 29, 34]. Nesse sentido, o ponto de partida para analisar um domínio de conhecimento é um caso [24].

De acordo com Spiro e Jehng [6], casos são coisas que realmente acontecem. Podem ser um caso médico, um evento histórico, uma notícia, um exemplo, uma situação em contexto [24, 28, 35]. Consistem em situações concretas em que se aplica o conhecimento conceitual [23]. A utilização de casos no ensino busca proporcionar uma aprendizagem contextualizada [21].

É importante esclarecer também que, nessa abordagem, a ideia não é deixar de ensinar conceitos. Tanto esses quanto os casos são essenciais. O que se altera é a forma com que os conhecimentos teóricos são trabalhados: no contexto de casos reais de sua aplicação, não no abstrato $[21,22]$. Em vez de construir conhecimento de exemplos, "o conhecimento é nos exemplos" [36, p. 96, tradução nossa, grifo do autor].

Cabe ressaltar também a sobrecarga cognitiva que a utilização dos casos pode impor na construção de conhecimentos, pelo fato de se constituírem em unidades complexas [34]. Tendo isso em vista, os autores da teoria enfatizam a necessidade de decompô-los em unidades menores, denominadas mini casos, de modo a explicitar os aspectos relevantes dos casos [6, 20].

Assim, o mini caso consiste na unidade fundamental de ensino [37], uma vez que a complexidade de um caso inviabiliza que ele desempenhe esse papel [20].

Os mini casos possibilitam abordar a complexidade a partir de pequenas unidades, de modo que aspectos que se dissipariam no todo passem a ter a sua importância [10, $21,23,28,34]$. Isso permite uma análise mais detalhada e uma melhor compreensão da situação [7].

Essa é a abordagem prevista na TFC: iniciar o estudo por conhecer o todo, o assunto na íntegra, para a partir daí analisar as partes que o constituem [29]. Assim, buscase tornar a complexidade cognitivamente gerenciável para o estudante. Em vez de partir de um tratamento simples para o complexo, o processo de ensino-aprendizagem inicia com tratamentos complexos, porém situados em mini 
casos cognitivamente gerenciáveis, auxiliando também na subsequente reestruturação do conhecimento $[6,20$, 37 .

Cabe destacar também que a utilização de mini casos assegura a flexibilidade necessária na análise dos casos pelo fato de possibilitar a consideração dos aspectos relevantes de cada caso e a identificação, em cada um desses aspectos, dos conceitos envolvidos [20], auxiliando ainda na ligação entre conceitos e casos [34].

Ao fornecer possíveis formas de análise de um caso [34], os mini casos permitem que aspectos relevantes de casos analisados anteriormente possam auxiliar no estudo de novas situações de aplicação de conhecimento. Nesse sentido, os mini casos constituem-se em uma das dimensões de flexibilidade presentes na TFC [20].

Realizada a decomposição dos casos complexos em mini casos com menor grau de complexidade, a próxima etapa consiste na análise desses mini casos [6]. Assim como os casos são analisados considerando seus aspectos relevantes - explicitados pelos mini casos -, cada mini caso também é analisado por meio dos diversos temas de análise conceitual que são relevantes para ele [6, 10, 29].

De acordo com os autores da TFC, temas de análise conceitual se referem a conceitos, princípios, leis e teorias, que variam de acordo com a área do saber [33]. Se constituem nos conhecimentos teóricos necessários para a compreensão dos mini casos e casos [23, 34]. Nesse sentido, os temas precisam ser ensinados em contexto e não de forma abstrata, de modo que a construção do significado desses seja influenciada pelas características do contexto de sua utilização [20].

Os temas desempenham, dessa forma, um papel fundamental, operando nos mini casos uma desconstrução essencial para a concretização de duas recomendações muito importantes da TFC: a utilização de múltiplas representações de conhecimento e de múltiplos esquemas interpretativos [20,32], que se constituem em dimensões de flexibilidade dessa teoria [34].

Realizada a seleção dos casos, mini casos e temas de análise conceitual, é preciso então efetuar a análise dos casos e mini casos através dos referidos temas. É nesse contexto que são produzidos o que os autores da TFC denominam comentários temáticos, que consistem em comentários que explicitam as diversas formas de aplicação de um determinado tema a uma dada situação (mini caso) $[6,7,10,20,24,34]$.

Uma vez realizada a desconstrução, estamos agora diante de um processo de reconstrução de conceitos. Pedro [20] aponta como contribuição dos comentários temáticos o fato de eles permitirem relacionar vários temas na mesma situação, ressaltando a importância da consideração de múltiplas perspectivas de análise. Nesse sentido, eles favorecem o entendimento do mini caso.

Já Pinho [24] cita que os comentários temáticos “[...] auxiliam o aluno a entender a relação de um dado tema com outros temas no mesmo mini caso, contextualizandoo" [24, p. 65]. Assim, eles contribuem também para a construção de estruturas conceituais flexíveis, visando sua reestruturação na análise de casos novos [20].

Dentro de um conjunto de casos, mini casos e temas de análise conceitual, é possível (e muito provável) que um mesmo tema não se aplique apenas a uma situação, mas sim esteja relacionado a diversos mini casos e casos. Nesse sentido, a consideração de um único contexto de aplicação de um determinado conteúdo pode limitar a construção flexível de conhecimentos.

Assim, para a operacionalização da TFC, há dois processos que os autores consideram essenciais e complementares, associados à desconstrução e reconstrução de conceitos $[7,8]$. Um deles possibilita a desconstrução do caso em mini casos analisados a partir dos temas associados, por meio dos comentários temáticos. O outro percurso permite atravessar diversos mini casos e comentários temáticos para compreender como um determinado tema se aplica em casos e mini casos distintos. Esse último processo é denominado travessia temática [10, 21, 23, 24, 27, 28]. Nesse sentido, a construção flexível de conhecimentos ocorre por meio da constante desconstrução e reconstrução de conceitos.

Nesse contexto, as travessias temáticas permitem explicitar - através dos comentários temáticos - como um mesmo tema se aplica a um conjunto heterogêneo de mini casos de diferentes casos [20, 24, 33].

Pode-se dizer, então, que a flexibilidade exigida em situações de aplicação de conhecimento é bidimensional: por um lado, os casos precisam ser decompostos em diversos mini casos; por outro lado, é preciso estabelecer diversas ligações entre os mini casos de diferentes casos, por meio dos comentários e travessias temáticas, aproximando mini casos distintos. Será essa travessia pelos diferentes mini casos que estabelecerá caminhos para a reconstrução do conhecimento para solucionar uma nova situação [9, 23, 24].

Ainda, as travessias temáticas contribuem para estabelecer diversas ligações entre casos e mini casos e conceitos, de modo a construir estruturas de conhecimento flexíveis que favoreçam a reestruturação das mesmas em novos contextos [20,32, 35, 37].

\section{Etapas para o Planejamento das $\mathrm{AD}$ de Física}

As AD de Física foram estruturadas com base nos pressupostos da TFC e mediadas por recursos educacionais hipermídia ${ }^{1}$, visando contribuir para consubstanciar os aspectos necessários para o desenvolvimento da FC em estudantes do ensino médio.

Para o planejamento dessas atividades, consideramos os aspectos necessários para o desenvolvimento da FC (explicitados na seção 2), bem como a estrutura principal

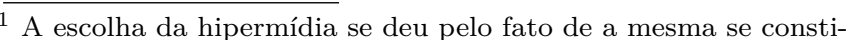
tuir no principal recurso didático para implementação da TFC conforme destacou Bolacha e Amador [26] e Leão, Souza e Moreira [38] -, contribuindo para promover características de FC [19]
} 
da TFC (caso, mini caso, tema de análise conceitual, comentário temático e travessia temática).

Anteriormente ao planejamento das AD, é importante explicitar que realizamos uma pesquisa bibliográfica em publicações de trinta e três periódicos das áreas de ensino e pesquisa em ensino de Física e Ciências, abrangendo o período de 1990 a 2017. O objetivo central foi, a partir da seleção e análise de artigos cuja ênfase estivesse na FC no referido âmbito, buscar subsídios para a elaboração e avaliação das AD de Física.

Obtivemos como resultado uma diversidade de metodologias de ensino, instrumentos de avaliação e focos de avaliação encontrada nas propostas analisadas. Conforme explicitaremos adiante, isso contribuiu significativamente para a estruturação de $\mathrm{AD}$ de Física que estivessem em consonância com os pressupostos teórico-metodológicos da TFC.

Nas subseções a seguir, apresentamos e discutimos as etapas envolvidas no planejamento das AD de Física.

\subsection{Seleção da temática a ser abordada}

$\mathrm{O}$ conjunto de $\mathrm{AD}$ aborda a energia elétrica nas residências, o que envolve os conteúdos de Física referentes à Eletrodinâmica. Essa temática consiste em parte de um todo maior, que busca estudar o caminho que a energia elétrica percorre desde sua geração até a tomada de nossas casas; ou seja, o processo de geração, transmissão e distribuição de energia elétrica.

A referida temática foi escolhida pois se constitui em uma temática complexa, que consiste no foco de aplicação da TFC [6, 21-24]. Isso porque diversos aspectos são necessários para a sua compreensão, além de envolver a aplicação prática de conhecimento [39], de modo que possibilita sua abordagem a partir de casos e mini casos $[6,23]$.

\subsection{Definição e sistematização dos casos, mini casos e temas de análise conceitual}

Realizada a seleção da temática a ser abordada, o próximo passo consistiu na definição e sistematização dos casos, mini casos e temas de análise conceitual, em consonância com a estrutura principal da TFC. O Quadro 1 apresenta os casos, mini casos e temas definidos.

De acordo com a TFC, os casos são situações concretas em que se aplica o conhecimento conceitual [23]. Essas situações precisam ser detentoras de complexidade, o que significa dizer elas requerem diversos conceitos para sua compreensão [19].

Os quatro casos foram escolhidos por se constituírem em aspectos relevantes a serem abordados quando se trata da energia elétrica nas residências. Desse modo, esses casos estão articulados entre si pela temática das AD.

Cabe aqui explicitar a principal dificuldade que encontramos na escolha dos casos, que foi a de definir o grau de complexidade adequado dos mesmos. Em um primeiro momento, escolhemos três casos que possuíam um grau de complexidade muito elevado, de modo que ao longo do processo de construção das AD constatamos a necessidade de reestruturá-los, aumentando a quantidade e diminuindo a complexidade dos mesmos.

Consideramos ainda a sugestão de Pedro [20] de que os casos não apresentem muitas semelhanças - prevenindo a formação de generalizações ilegítimas -, mas também não apresentem tantas diferenças, o que tornaria difícil a percepção da existência de semelhanças.

Nesse sentido, os casos escolhidos apresentam semelhanças, na medida em que são analisados com alguns aspectos semelhantes (mini casos que abordam as causas e consequências, bem como as formas de evitar, tanto do curto-circuito quanto do choque elétrico); mas também diferenças, uma vez que sua compreensão também envolve aspectos distintos.

É importante mencionar também que os quatro casos permitem abranger diferentes perspectivas do conceito, o que auxilia na sua compreensão e aplicação em diferentes situações concretas, conforme destacaram Souza e Leão [32].

Em relação aos mini casos, os autores da TFC afirmam que eles explicitam os aspectos relevantes dos casos [6,

Quadro 1: Casos, mini casos e temas de análise conceitual das AD.

\begin{tabular}{|c|c|c|}
\hline Casos & Mini casos & Temas de análise conceitual \\
\hline $\begin{array}{l}\text { Caso 1: ligação do circuito } \\
\text { elétrico nas residências }\end{array}$ & $\begin{array}{l}\text { Mini caso 1.1: mono, bi e trifásico } \\
\text { Mini caso } 1.2: 127 \mathrm{~V} \text { e } 220 \mathrm{~V} \\
\text { Mini caso } 1.3: \text { ligação dos aparelhos elétricos }\end{array}$ & $\begin{array}{l}\text { corrente elétrica, frequência, lei de conserva- } \\
\text { ção de energia, lei de Ohm, potência elétrica, } \\
\text { resistência elétrica, tensão elétrica }\end{array}$ \\
\hline $\begin{array}{l}\text { Caso 2: consumo de energia } \\
\text { elétrica }\end{array}$ & $\begin{array}{l}\text { Mini caso 2.1: características dos aparelhos elétri- } \\
\text { cos } \\
\text { Mini caso 2.2: lâmpadas } \\
\text { Mini caso 2.3: conta de luz }\end{array}$ & $\begin{array}{l}\text { corrente elétrica, efeito Joule, energia elétrica, } \\
\text { frequência, lei de conservação de energia, lei de } \\
\text { Ohm, } \\
\text { potência elétrica, resistência elétrica, } \\
\text { tensão elétrica }\end{array}$ \\
\hline Caso 3: curto-circuito & $\begin{array}{l}\text { Mini caso 3.1: causas e consequências } \\
\text { Mini caso 3.2: adequação dos fios } \\
\text { Mini caso 3.3: importância do fusível e disjuntor }\end{array}$ & $\begin{array}{l}\text { corrente elétrica, efeito Joule, lei de Ohm, po- } \\
\text { tência elétrica, resistência elétrica, tensão elé- } \\
\text { trica }\end{array}$ \\
\hline Caso 4: choque elétrico & $\begin{array}{l}\text { Mini caso 4.1: causas e consequências } \\
\text { Mini caso } 4.2 \text { : adequação das tomadas } \\
\text { Mini caso } 4.3 \text { : importância do aterramento }\end{array}$ & $\begin{array}{l}\text { corrente elétrica, lei de } \mathrm{Ohm} \text {, potência elétrica, } \\
\text { resistência elétrica, tensão elétrica }\end{array}$ \\
\hline
\end{tabular}


20], permitindo, assim, uma análise mais detalhada e uma melhor compreensão da situação [7].

Nesse sentido, considerando a abrangência dos quatro casos, definimos doze mini casos (três para cada caso) que nos possibilitassem abordar os aspectos que consideramos relevantes dentro de cada caso, além de identificar, em cada um desses aspectos, os conceitos envolvidos. Desse modo, os mini casos estão articulados entre si pelos casos e conceitos.

Cabe aqui explicitar que a principal dificuldade que encontramos na escolha dos mini casos foi a de definir a quantidade adequada, de modo a considerarmos apenas aqueles que nos permitissem abordar aspectos que fossem de fato relevantes para cada caso. Ao longo do processo de construção das $\mathrm{AD}$, os mini casos foram reestruturados diversas vezes, uma vez que constatamos a necessidade de reduzir a quantidade inicialmente escolhida.

Para auxiliar na escolha dos mini casos, consideramos o esclarecimento de Spiro e Jehng [6] de que esses não são partes abstratas de um caso. Ou seja, que a característica de concretude dos casos está presente também nos mini casos; de modo que, através deles, os conceitos não são abordados de forma abstrata, mas sim de forma contextualizada [20].

Além disso, levamos em conta que os mini casos precisam ser suficientemente pequenos para permitirem uma análise rápida num tempo letivo, mas suficientemente complexos para serem analisados com diversos conceitos, conforme salientou Carvalho [23].

Assim como os casos são analisados considerando seus aspectos relevantes - explicitados pelos mini casos -, cada mini caso também é analisado por meio dos diversos temas de análise conceitual que são relevantes para ele $[6,10]$. De acordo com os autores da TFC, temas de análise conceitual se referem a conceitos, princípios, leis e teorias; ou seja, aos conhecimentos teóricos necessários para a compreensão dos mini casos e casos [21, 23, 34].

Nesse sentido, definimos nove temas de análise conceitual que nos possibilitassem compreender os doze mini casos e os quatro casos escolhidos. Desse modo, os temas estão articulados entre si pelos casos e mini casos.
Em relação à escolha desses temas, Pedro [20] afirma que, muitas vezes, é difícil determinar, a partir de um caso ou mini caso, os conceitos relevantes nessa situação e, inversamente, é difícil ajustar um conceito às características particulares das suas situações de aplicação. Essa dificuldade esteve presente na definição dos nove temas.

Assim como ocorreu com os mini casos, a principal dificuldade foi definir a quantidade adequada temas, optando apenas por aqueles que de fato fossem relevantes para a compreensão dos casos e mini casos. Ao longo do processo de construção das $\mathrm{AD}$, reduzimos o número de temas a aproximadamente metade da quantidade inicial.

Para a sistematização dos casos, mini casos e temas de análise conceitual, construímos uma Matriz Temática - sugerida pelos autores da TFC e apresentada por Carvalho [23] - composta pelos casos, mini casos e temas. Ela permite visualizar os casos e mini casos que têm afinidades temáticas, ou seja, que são analisados pelos mesmos temas. O Quadro 2 apresenta a referida matriz.

Os casos (1 a 4) e os mini casos (1.1 a 4.3) contidos no Quadro 2 são aqueles explicitados no Quadro 1. Já os temas de análise conceitual (A a I) contidos no Quadro 2 são resultantes da junção daqueles explicitados no Quadro 1, denominados da seguinte forma: A. corrente elétrica; B. efeito Joule; C. energia elétrica; D. frequência; E. lei de conservação de energia; F. lei de Ohm; G. potência elétrica; H. resistência elétrica; I. tensão elétrica.

Analisando a matriz, observamos que cada tema possui maior aplicabilidade em alguns mini casos do que em outros, conforme salientado por Carvalho et al. [33], resultando no fato de que alguns temas se aplicam a um número maior de mini casos do que outros.

Em relação aos mini casos, notamos que alguns deles são analisados através de uma quantidade maior de temas do que outros. Entretanto, nenhum deles está associado a apenas um tema, o que poderia restringir a compreensão dos mesmos, conforme destacou Carvalho [23].

Como exemplo de leitura vertical da matriz, visualizamos que o tema resistência elétrica (tema $\mathrm{H}$ ) contribui para compreender: como se dá a ligação dos aparelhos elétricos ao circuito nas residências (mini caso 1.3); as

Quadro 2: Matriz Temática para as AD de Física.

\begin{tabular}{|c|c|c|c|c|c|c|c|c|c|c|}
\hline & & & & tas & le & ális & $\mathrm{co}$ & cei & & \\
\hline Caso & Mini caso & $\mathbf{A}$ & $\mathbf{B}$ & $\mathbf{C}$ & D & $\mathbf{E}$ & $\mathbf{F}$ & G & $\mathbf{H}$ & $\mathbf{I}$ \\
\hline \multirow{3}{*}{1} & 1.1 & $\mathrm{X}$ & & & & $\mathrm{X}$ & & $\mathrm{X}$ & & $\mathrm{X}$ \\
\hline & 1.2 & $\mathrm{X}$ & & & & & $\mathrm{X}$ & $\mathrm{X}$ & & $\mathrm{X}$ \\
\hline & 1.3 & $\mathrm{X}$ & & & $\mathrm{X}$ & & $\mathrm{X}$ & & $\mathrm{X}$ & $\mathrm{X}$ \\
\hline \multirow{3}{*}{2} & 2.1 & $\mathrm{X}$ & $\mathrm{X}$ & & $\mathrm{X}$ & & $\mathrm{X}$ & $\mathrm{X}$ & $\mathrm{X}$ & $\mathrm{X}$ \\
\hline & 2.2 & $\mathrm{X}$ & $\mathrm{X}$ & & $\mathrm{X}$ & & $\mathrm{X}$ & $\mathrm{X}$ & $\mathrm{X}$ & $\mathrm{X}$ \\
\hline & 2.3 & & & $\mathrm{X}$ & & $\mathrm{X}$ & & $\mathrm{X}$ & & \\
\hline \multirow{3}{*}{3} & 3.1 & $\mathrm{X}$ & $\mathrm{X}$ & & & & $\mathrm{X}$ & & $\mathrm{X}$ & \\
\hline & 3.2 & $\mathrm{X}$ & $\mathrm{X}$ & & & & $\mathrm{X}$ & $\mathrm{X}$ & $\mathrm{X}$ & $\mathrm{X}$ \\
\hline & 3.3 & $\mathrm{X}$ & $\mathrm{X}$ & & & & $\mathrm{X}$ & $\mathrm{X}$ & & $\mathrm{X}$ \\
\hline \multirow{3}{*}{4} & 4.1 & $\mathrm{X}$ & & & & & $\mathrm{X}$ & & $\mathrm{X}$ & $\mathrm{X}$ \\
\hline & 4.2 & $\mathrm{X}$ & & & & & & & & $\mathrm{X}$ \\
\hline & 4.3 & $\mathrm{X}$ & & & & & $\mathrm{X}$ & & $\mathrm{X}$ & \\
\hline
\end{tabular}


características dos aparelhos elétricos (mini caso 2.1); as lâmpadas (mini caso 2.2); as causas e consequências do curto-circuito (mini caso 3.1); a adequação dos fios para evitar o curto-circuito (mini caso 3.2); as causas e consequências do choque elétrico (mini caso 4.1); e a importância do aterramento para evitar o choque elétrico (mini caso 4.3).

Já como exemplo de leitura horizontal da matriz, visualizamos que a compreensão da importância do fusível e do disjuntor para evitar o curto-circuito (mini caso 3.3) envolve os seguintes temas de análise conceitual: corrente elétrica (tema A); efeito Joule (tema B); lei de Ohm (tema $\mathrm{F}$ ); potência elétrica (tema $\mathrm{G}$ ) e tensão elétrica (tema I).

Assim, por meio dessa sistematização, podemos visualizar possibilidades de comentários temáticos e de travessias temáticas a serem explorados nas AD de Física, em consonância com a estrutura da TFC.

A matriz contribui também para concretizar um dos aspectos envolvidos nas múltiplas representações do conhecimento: o das múltiplas formas de organização do conhecimento, através de casos, mini casos e temas de análise conceitual interligados [19, 31, 32], essencial para o desenvolvimento da FC.

Além disso, a matriz também auxilia na consubstanciação de outros aspectos necessários para o desenvolvimento da FC, a saber: desconstrução de casos e mini casos por meio de diferentes temas; estabelecimento de ligações entre mini casos de diferentes casos; e promoção de diversas ligações entre conceitos e casos [21-23, 30].

\subsection{Consulta a materiais}

De modo a auxiliar no processo de planejamento das AD, consultamos diversos materiais. O critério e a estratégia utilizada para a busca estiveram em consonância com a estrutura da TFC. Nesse sentido, nossa pesquisa esteve focada em repositórios de materiais didáticos de Física nos moldes da hipermídia (imagens, gráficos, áudios, vídeos, animações, simulações computacionais), bem como portais que nos auxiliassem na operacionalização dos casos e mini casos descritos acima.

Assim, os materiais foram advindos das seguintes fontes:

- Notícias de jornais eletrônicos;

- Portal da Agência Nacional de Energia Elétrica $(\mathrm{ANEEL}){ }^{2}$

- Portal de Geradoras, Transmissoras e/ou Distribuidoras de energia elétrica;

- Livros didáticos de Física do ensino médio e do ensino superior;

- Portal do Centro de Referência para o Ensino de Física $(\mathrm{CREF})^{3}$

- Portal do Professor 4

\footnotetext{
2 http://www.aneel.gov.br/

3 https://www.if.ufrgs.br/novocref/

${ }^{4}$ http://portaldoprofessor.mec.gov.br/index.html
}

- Banco Internacional de Objetos Educacionais ${ }^{5}$

- Portal Physics Education Tecnology (PhET)

- Multimedia Educational Resource for Learning and Online Teaching (MERLOT) ${ }^{7}$

- ComPADRE8.

- Portal Física Vivencia ${ }^{9}$

- Portal Tópicos de Ciência e Tecnologia Contemporâneas 10

- Portal Applets Java de Física ${ }^{11}$

- Portal Dia a Dia Educaçãd ${ }^{12}$

- Laboratório Didático Virtual (LabVirt) 13

- Coleção francesa de vídeos didáticos Viagem na Eletricidade ${ }^{14}$.

- Mundo de Beakman 15

- Provas do Exame Nacional do Ensino Médio (ENEM)

\subsection{Definição dos objetivos gerais e específicos}

O conjunto de $\mathrm{AD}$ de Física possui um objetivo formativo mais amplo, para além do conhecimento conceitual, que diz respeito a uma compreensão de mundo articulada por parte do sujeito. É esse objetivo mais amplo que gera a necessidade do desenvolvimento da FC.

Nesse sentido, as seis AD possuem três objetivos gerais comuns - que estão em consonância com os objetivos da TFC - a partir dos quais elas estão estruturadas. São eles:

- construir flexivelmente os conceitos físicos 17

- compreender e relacionar adequadamente os conceitos físicos ${ }^{17}$;

- aplicar adequadamente os conceitos físicos na resolução de problemas e/ou situações-problema ${ }^{18}$.

Conforme mencionamos, a TFC é voltada à abordagem de conhecimentos de nível avançado em domínios pouco estruturados. Cabe aqui destacar que as AD de Física planejadas estão em consonância com esse contexto, na medida em que: (i) o objetivo principal das mesmas é que os estudantes do ensino médio compreendam profundamente os conceitos físicos envolvidos e os apliquem em situações novas, em conformidade com os objetivos de aprendizagem dos conhecimentos de nível avançado; e (ii) abordam a aplicação dos conhecimento físicos em si-

\footnotetext{
5 http://objetoseducacionais2.mec.gov.br

6 http://phet.colorado.edu/pt_BR/

7 http://wWw.merlot.org/merlot/index.htm

8 http://www.compadre.org/

9 http://www.fisicavivencial.pro.br/

10 http://www.ced.ufsc.br/men5185/

11 https://www.walter-fendt.de/html5/phpt/

12 http://www.diaadia.pr.gov.br

13 http://WwW.labvirt.fe.usp.br/institucional . asp?time=15: $26: 59$

${ }_{14}^{26.59}$ https : //www . youtube. com/playlist?list=PLYfrhgvQ39rW_ WIYQgEK04nr5rSz1rgGP

1 https://www youtube $\cdot$ com/watch?v=cJOycnaaHVg $\{\&\}$ list= PL110SyHHXtuL91tPDP34q1X4NGGqsr4Ri

10 http://portal.inep.gov.br/web/guest/provas-e-gabaritos

17 Esses objetivos consistem em aspectos necessários para o desenvolvimento da FC.

18 Esse objetivo diz respeito à recontextualização de conhecimento para novas situações.
} 
tuações concretas, trazendo ao domínio de conhecimento aspectos de pouca estruturação.

Além dos objetivos gerais, cada AD possui objetivos específicos, articulados aos objetivos gerais, que consideram os caso(s), mini caso(s) e temas de análise conceitual específicos de cada uma.

\subsection{Estruturação das AD}

As AD também possuem uma estrutura geral comum, que está em consonância com elementos que a TFC apresenta para consubstanciar os aspectos necessários para o desenvolvimento da FC: caso, mini caso, tema de análise conceitual, comentário temático e travessia temática.

Entretanto, mesmo estando presente na estrutura geral das atividades, optamos por não utilizar a nomenclatura desses elementos tal qual ela consta na TFC, por supormos que os estudantes do ensino médio não tem conhecimento da referida teoria, o que no nosso entendimento poderia causar certa confusão.

Nesse sentido, o conjunto de AD possui a seguinte estrutura geral:

$\rightarrow$ Situação-problema inicial. Situação-problema consiste em um problema relacionado a uma situação concreta cuja compreensão envolve conhecimentos teóricos.

Nesse sentido, elaboramos uma situação-problema para iniciar cada uma das atividades. Essa situação-problema está articulada ao(s) mini caso(s) e ao caso a serem abordados e, em alguns casos, foi elaborada a partir de uma notícia publicada que possuía vínculo com o(s) mini caso(s) e o caso.

Essa estratégia de ensino está em consonância com a TFC na medida em que esta possui uma abordagem centrada no estudo de casos [23, 34], que constituem o ponto de partida [24]. Estreitamente associados aos casos, estão os mini casos, que explicitam seus aspectos relevantes [6].

Com isso, buscamos enfatizar os seguintes aspectos necessários para o desenvolvimento da FC: concepção do conhecimento conceitual como conhecimento em utilização, de modo que o significado de um conceito está vinculado ao seu contexto de aplicação; e enfoque no caso como unidade de análise dos domínios de conhecimento.

Cabe explicitar a dificuldade que encontramos em algumas AD de elaborar uma situação-problema que abrangesse o(s) mini caso(s) e o caso a serem abordados. Consideramos esse como o ponto chave para uma definição adequada da mesma.

$\rightarrow$ Conteúdos de Física. Abordagem dos conceitos, princípios, leis e teorias de Física necessários para a compreensão e resolução da situação-problema inicial.

Para auxiliar nesse processo, optamos pela utilização de recursos didáticos em diversas mídias, tais como textos, imagens, vídeos, animações e/ou simulações. A partir desse material, consideramos essencial a abordagem dos conteúdos ser realizada pelo professor utilizando tam- bém o quadro, no qual são discutidos com os estudantes definições, equações e unidades de medida das grandezas físicas envolvidas. Além disso, as AD possuem links para os conceitos tensão, corrente, resistência e potência elétrica, que direcionam a textos que trabalham esses conceitos. Cabe sublinhar que essa interseção entre a multimídia e o hipertexto caracteriza a hipermídia.

Essa estratégia de ensino está em consonância com a TFC na medida em que, uma vez realizado o processo de desconstrução dos casos em mini casos, há um novo processo de desconstrução, agora dos mini casos em temas de análise conceitual, que dizem respeito aos conhecimentos teóricos necessários para a compreensão dos mini casos e casos [6, 29].

Com essa etapa da AD, buscamos enfatizar os seguintes aspectos necessários para o desenvolvimento da FC: existência de múltiplas representações do conhecimento; e desconstrução de casos e mini casos através de diferentes temas.

$\rightarrow$ Resolução de problemas. Proposição de problemas a serem resolvidos pelo estudante, utilizando os conteúdos de Física abordados.

Os problemas estão articulados entre si pela situaçãoproblema inicial e pelo(s) mini caso(s) e caso, e buscam abordar aspectos relevantes dos mesmos.

Optamos por utilizar problemas fechados, abertos, qualitativos e quantitativos, abrangendo a diversidade que consideramos necessária para uma melhor compreensão da situação-problema inicial e, com isso, do(s) mini caso(s) e caso associados.

Juntamente com os problemas, são disponibilizados materiais em diversas mídias - tais como textos, imagens, vídeos e simulações computacionais - e acessados por meio de diversos links, para auxiliar no processo de compreensão e resolução dos mesmos. Assim como na etapa anterior, temos aqui materiais organizados nos moldes da hipermídia.

No âmbito da TFC, uma vez realizado o processo de desconstrução, se dá o processo de reconstrução por meio do comentário temático, no qual é explicitado de que forma cada tema de análise conceitual se aplica para a compreensão de um determinado mini caso [6, 7, 10, 34].

É nessa etapa da $\mathrm{AD}$ que o estudante terá que utilizar os conteúdos de Física para resolver os problemas. Isso significa, no âmbito da TFC, explicitar como cada tema de análise conceitual se aplica na compreensão do mini caso, caracterizando o comentário temático.

Com essa etapa da AD, buscamos enfatizar os seguintes aspectos necessários para o desenvolvimento da FC: existência de múltiplas representações do conhecimento; concepção do conhecimento conceitual como conhecimento em utilização, de modo que o significado de um conceito está vinculado ao seu contexto de aplicação; construção flexível de conhecimento; e promoção de diversas ligações entre conceitos e casos.

Consideramos como ponto chave nesta etapa a elaboração de problemas em quantidade e abrangência adequados, que contribuam para a compreensão da situação- 
problema inicial, bem como do(s) mini caso(s) e caso associados.

$\rightarrow$ Análise da situação-problema. Retomada pelo estudante da situação-problema proposta inicialmente.

Nesta etapa da AD, a situação-problema inicial é novamente apresentada para que o estudante analise-a e responda-a, considerando os conteúdos de Física estudados e os problemas por ele resolvidos.

Assim como na etapa anterior, aqui ocorre o processo de reconstrução através do comentário temático. Em relação aos aspectos necessários para o desenvolvimento da FC, além daqueles abordados na etapa anterior, destacamos a contribuição dessa etapa no sentido de possibilitar ao estudante relacionar, por meio dos comentários temáticos, um dado tema com outros temas no mesmo mini caso, favorecendo a construção flexível do conhecimento e a compreensão do mini caso.

$\rightarrow$ Síntese. Realização de uma síntese do que foi abordado na AD.

Esta etapa consiste na realização, pelo professor, de uma síntese dos conteúdos de Física abordados, da situaçãoproblema e da vinculação entre ambos. Optamos em algumas $\mathrm{AD}$ por utilizar imagens e/ou vídeos que, no nosso entendimento, auxiliam nessa síntese.

O objetivo aqui é retomar os principais aspectos trabalhados ao longo da atividade, em termos de conteúdos de Física, da situação-problema e de como esses se articulam. No âmbito da TFC, a ideia é retomar os principais temas de análise conceitual, explicitando como eles se aplicam na compreensão do(s) mini caso(s) e do caso.

Em relação aos aspectos necessários para o desenvolvimento da FC, destacamos, para essa etapa da $\mathrm{AD}$ : as múltiplas representações do conhecimento; e a promoção de diversas ligações entre conceitos e casos.

$\rightarrow$ Material extra. Sugestão de leituras complementares para casa.

Denominado "Saiba mais", esse material é composto por textos, perguntas e respostas, reportagens, imagens e vídeos. O objetivo é proporcionar ao estudante o aprofundamento dos principais aspectos abordados na $\mathrm{AD}$, de acordo com o interesse de cada um.

$\rightarrow$ Aplicação do conhecimento. Proposição de novos problemas e/ou situações-problema a serem resolvidos pelo estudante.

Para finalizar a AD, são propostos novos problemas e/ou situações-problema relacionados ao que foi abordado até então (situação-problema inicial, conteúdos de Física e problemas). Em algumas atividades são utilizados textos, imagens e links para outros sites, de modo a auxiliar na ilustração, compreensão e análise.

O objetivo nessa etapa é que o estudante aplique o que foi abordado para compreender novas situações e contextos, denominado pelos autores da TFC por recontextualização do conhecimento.

Consideramos importante explicitar que a nossa principal dificuldade no processo de elaboração das AD foi elaborar situações novas que, de fato, envolvessem a recontextualização de conhecimentos.
De acordo com a TFC, uma situação nova de aplicação de conhecimento envolve a combinação de diversos conceitos [20], podendo estar associada, por exemplo, a um problema a ser resolvido [6, 23]. O aspecto central na identificação dessa situação nova é que nela precisa haver características que a diferem da situação de aprendizagem [21].

Mesmo com esses esclarecimentos, nossa dificuldade foi definir com clareza quão diferente das situações anteriores precisa ser essa nova situação; qual a "distância" adequada entre elas.

Nesse contexto, optamos pelo equilíbrio; ou seja, por elaborar situações que não apresentassem muitas semelhanças com as anteriores (o que no nosso entendimento não privilegiaria a recontextualização do conhecimento) e nem muitas diferenças (o que no nosso entendimento tornaria muito difícil a percepção da existência de semelhanças e, com isso, a recontextualização do conhecimento).

O Quadro 3 sistematiza a estrutura geral das $\mathrm{AD}$, bem como sua relação com os aspectos da TFC.

\section{AD de Física Planejadas: Energia Elétrica nas Residências}

O conjunto é composto por seis $\mathrm{AD}$ que abordam os referidos casos e mini casos, de acordo com a organização apresentada no Quadro 4.

As AD estão disponíveis no site http://www.ufsm. $\mathrm{br} / \mathrm{mpeac} / \mathrm{muryel}$, que torna possível a integração das tecnologias da informação e comunicação - no nosso caso, mais especificamente, da hipermídia - nessas atividades. Cada uma delas contém o material do professor e o material do estudante.

No material do professor a AD precisa estar descrita de forma detalhada, de modo que qualquer professor ou futuro professor que tiver acesso a esse material tenha condições de acompanhar, ao longo da leitura, a intencionalidade dos autores com cada etapa da atividade. Esse material precisa estar claramente relacionado ao material do estudante.

Já o material do estudante serve como um roteiro para a realização da $\mathrm{AD}$, sendo composto de tarefas a serem apresentadas durante a atividade. Desse modo, esse material consiste em um registro das atividades que os estudantes terão de realizar, que pode ser entregue a eles de uma vez ou exposto aos poucos durante a realização da AD.

A seguir, apresentamos de forma sucinta, as seis $\mathrm{AD}$, voltadas a $3^{\mathrm{a}}$ série do ensino médio. O material completo das atividades pode ser acessado por meio do link https://drive.google.com/open?id= 1udd0c9dET5F68P8NaPhGv8DAxkXi3IcC 
Quadro 3: Estrutura geral das AD e os aspectos da TFC.

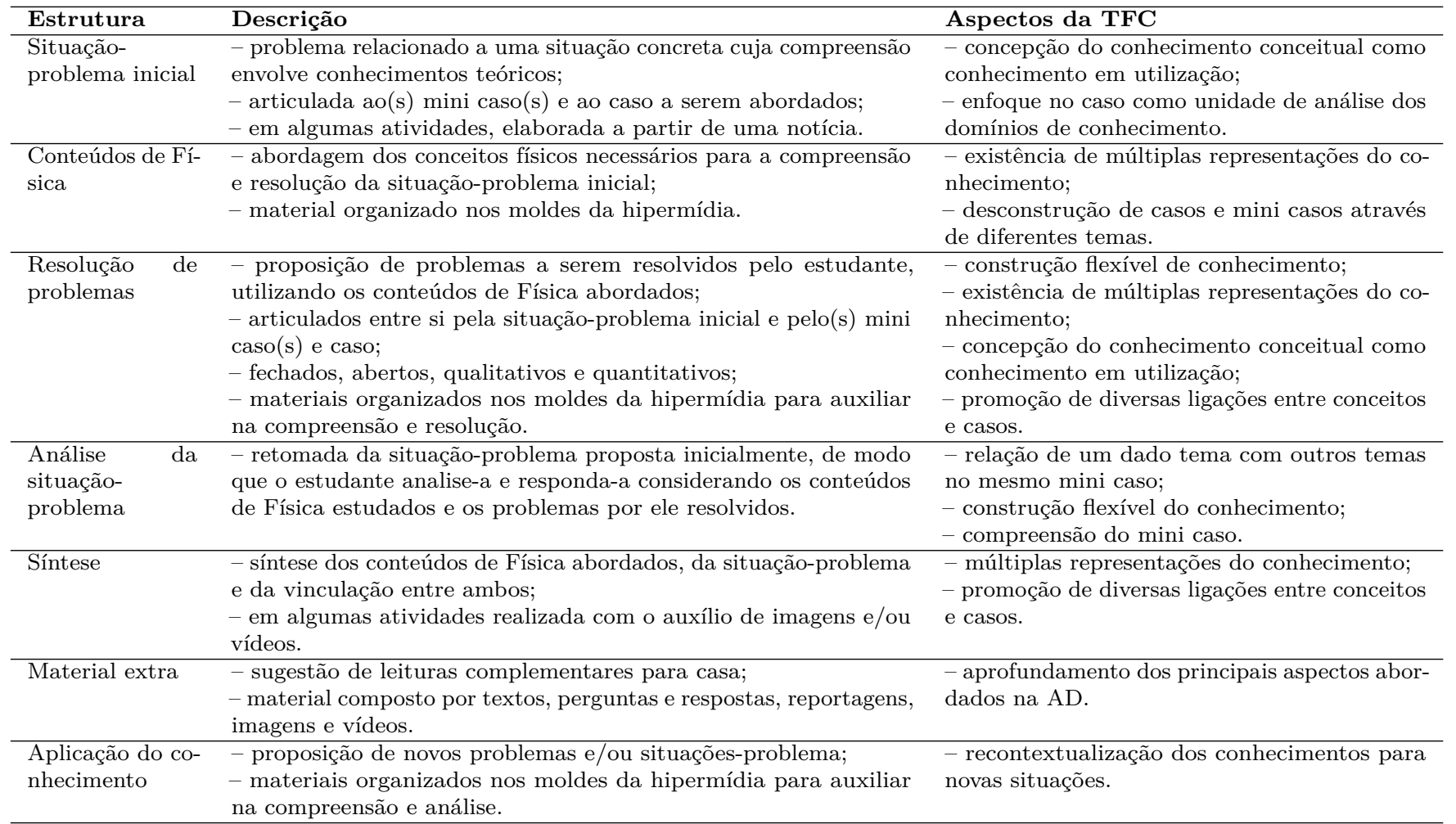

Quadro 4: Organização geral das AD.

\begin{tabular}{lcc}
\hline AD & Caso & Mini caso \\
\hline 1 & 1 & $1.1 \mathrm{e} 1.2$ \\
2 & & 1.3 \\
3 & 2 & $2.1 \mathrm{e} 2.2$ \\
4 & 2.3 \\
5 & 3 & $3.1,3.2 \mathrm{e} 3.3$ \\
6 & 4 & $4.1,4.2 \mathrm{e} 4.3$ \\
\hline
\end{tabular}

\subsection{AD 1 - Ligação do circuito elétrico nas residências}

A AD 1 aborda as ligações mono, bi e trifásica (mini caso 1.1) e $127 \mathrm{~V}$ e $220 \mathrm{~V}$ (mini caso 1.2), como parte da ligação do circuito elétrico nas residências (caso 1). Os temas de análise conceitual envolvidos são: corrente elétrica, lei de conservação de energia, lei de Ohm, potência elétrica e tensão elétrica.

Essa AD tem como objetivos específicos: i) vincular a tensão elétrica fornecida às residências à diferença de potencial entre o fase e o neutro; ii) vincular a presença da corrente elétrica em um circuito ao fato de o mesmo estar fechado; iii) associar adequadamente os conceitos de tensão e corrente elétrica; iv) identificar as características e diferenças das ligações monofásicas, bifásicas e trifásicas; v) identificar as características e diferenças das ligações $127 \mathrm{~V}$ e $220 \mathrm{~V}$; vi) aplicar adequadamente os conceitos físicos abordados para compreender como se dá a ligação do circuito elétrico nas residências.
Como recursos didáticos, são utilizados reportagens, textos de livros didáticos, textos de sites, resolução de problemas, imagens e vídeos.

Para a realização da $\mathrm{AD}$, serão necessários três aulas (com cinquenta minutos cada). A atividade está estruturada em três etapas, e tem como ponto de partida a seguinte situação-problema: como é a ligação do circuito elétrico nas residências?

A primeira etapa aborda as características e diferenças das ligações monofásicas, bifásicas e trifásicas. Na segunda etapa, são abordadas as características e diferenças da utilização da tensão 127 V e 220 V. Por fim, na terceira etapa, os estudantes resolverão individualmente duas situações associadas ao conteúdo abordado nas etapas anteriores.

\subsection{AD 2 - Ligação dos aparelhos elétricos ao circuito}

A AD 2 dá sequência ao estudo da ligação do circuito elétrico nas residências (caso 1), abordando a abordando a ligação dos aparelhos elétricos (mini caso 1.3). Os temas de análise conceitual envolvidos são: corrente elétrica, frequência, lei de Ohm, potência elétrica, resistência elétrica e tensão elétrica.

Essa AD tem como objetivos específicos: i) associar a frequência da energia elétrica fornecida ao funcionamento dos aparelhos elétricos; ii) vincular o aumento da corrente elétrica nos circuitos ao aumento do consumo de energia 
elétrica; iii) compreender a função do interruptor em um circuito elétrico; iv) identificar os fatores pelos quais a ligação do circuito nas residências se dá em paralelo e não em série; v) aplicar adequadamente os conceitos físicos abordados para compreender como se dá a ligação dos aparelhos elétricos ao circuito nas residências.

Como recursos didáticos, são utilizados textos de livros didáticos, resolução de problemas, imagens, vídeos e simulação computacional.

Para a realização da $\mathrm{AD}$, serão necessários quatro aulas (com cinquenta minutos cada). A atividade está estruturada em três etapas, e tem como ponto de partida a seguinte situação-problema: como é a ligação dos aparelhos elétricos ao circuito nas residências?

A primeira etapa aborda as características e diferenças das ligações série e paralelo, de modo a explicitar os fatores pelos quais a ligação dos aparelhos nas residências se dá em paralelo e não em série. Já a segunda etapa consiste na realização de uma síntese do que foi abordado, utilizando para isso uma imagem e um vídeo. Para finalizar a atividade, na terceira etapa, os estudantes resolverão individualmente alguns problemas do Enem, buscando aplicar o conhecimento em diferentes situações.

\subsection{AD 3 - Aparelhos elétricos e lâmpadas}

Dando início ao estudo do caso 2, consumo de energia elétrica, a AD 3 aborda as características dos aparelhos elétricos (mini caso 2.1) e lâmpadas (mini caso 2.2). Os temas de análise conceitual envolvidos são: corrente elétrica, efeito Joule, frequência, lei de Ohm, potência elétrica, resistência elétrica, tensão elétrica.

Essa AD tem como objetivos específicos: i) identificar os valores nominais de potência, corrente, tensão e resistência elétrica de um aparelho elétrico; ii) relacionar adequadamente potência, corrente, tensão e resistência elétrica de um aparelho elétrico; iii) verificar a relação de dependência entre o consumo de energia elétrica de um aparelho e a tensão da rede a qual ele está ligado; iv) aplicar adequadamente os conceitos físicos abordados para compreender o que acontece ao ligarmos um aparelho elétrico em uma rede cuja tensão é diferente da nominal; v) aplicar adequadamente os conceitos físicos abordados para analisar que tipo de lâmpada (incandescente, fluorescente compacta ou LED) é mais vantajosa em termos de consumo de energia elétrica e financeiramente.

Como recursos didáticos, são utilizados notícias, reportagens, textos de livros didáticos, textos de sites, catálogo de fabricantes de lâmpadas, resolução de problemas, imagens e vídeos.

Para a realização da $\mathrm{AD}$, serão necessários quatro aulas (com cinquenta minutos cada). A atividade está estruturada em cinco etapas, e tem como ponto de partida a seguinte situação-problema: O que acontece ao ligarmos um aparelho elétrico fabricado para $220 \mathrm{~V}$ em uma rede elétrica de $127 \mathrm{~V}$, e vice-versa?

$\mathrm{Na}$ primeira etapa, os estudantes elaborarão uma resposta inicial à situação-problema a partir dos conheci- mentos que possuem. A segunda etapa consiste na identificação, pelos estudantes, dos valores nominais de um aparelho elétrico escolhido por eles, seguido pela resolução de problemas sobre o assunto. Na terceira etapa, serão trabalhadas especificamente as lâmpadas, através da resolução de problemas e leitura de textos. Já na quarta etapa, os estudantes responderão novamente a questão inicial, considerando o que foi trabalhado na atividade. Por fim, na quinta etapa, os estudantes resolverão individualmente duas situações associadas ao conteúdo abordado nas etapas anteriores.

\subsection{AD 4 - Entendendo a conta de luz}

A AD 4 dá continuidade ao estudo do caso 2, consumo de energia elétrica, abordando especificamente a conta de luz (mini caso 2.3). Os temas de análise conceitual envolvidos são: energia elétrica, lei de conservação de energia e potência elétrica.

Essa AD tem como objetivos específicos: i) identificar os elementos que constituem o valor da conta de luz; ii) estimar o consumo mensal de energia elétrica em sua residência; iii) identificar ações que resultam em uma redução no consumo de energia elétrica em sua residência; iv) aplicar adequadamente os conceitos físicos abordados para compreender os fatores que interferem no consumo de energia elétrica.

Como recursos didáticos, são utilizados textos de livros didáticos, textos de sites, notícias, resolução de problemas, imagens, vídeos e simuladores de consumo de energia elétrica.

Para a realização da $\mathrm{AD}$, serão necessários quatro aulas (com cinquenta minutos cada). A atividade está estruturada em cinco etapas, e tem como ponto de partida a seguinte situação-problema: uma lâmpada pode consumir mais energia elétrica do que um chuveiro?

A primeira etapa inicia com a leitura de uma notícia sobre o consumo da energia elétrica, seguida da análise inicial da referida situação-problema pelos estudantes. $\mathrm{Na}$ segunda etapa, os estudantes realizarão a leitura de dois textos sobre medida de energia elétrica na residência, seguida pela ilustração de um vídeo e pela resolução de problemas sobre o assunto. Na terceira etapa, os estudantes farão a leitura de dois textos que abordam os componentes da conta de luz e o consumo de stand by, além de resolver problemas sobre isso. Já a quarta etapa consiste em os estudantes responderem novamente a questão inicial, considerando o que foi trabalhado na atividade. Por fim, na quinta etapa, os estudantes resolverão individualmente três situações associadas ao conteúdo abordado nas etapas anteriores.

\subsection{AD 5 - Curto-circuito}

A AD 5 aborda o estudo do curto-circuito (caso 3), especificamente as causas e consequências (mini caso 3.1), a adequação dos fios (mini caso 3.2) e a importância do fusível e disjuntor (mini caso 3.3). Os temas de análise conceitual envolvidos são: corrente elétrica, efeito Joule, 
lei de Ohm, potência elétrica, resistência elétrica e tensão elétrica.

Essa AD tem como objetivos específicos: i) relacionar adequadamente os conceitos de tensão, resistência, corrente e potência elétrica; ii) identificar as causas e consequências do curto-circuito na rede elétrica; iii) perceber a adequação dos fios como um fator determinante para evitar o curto-circuito; iv) analisar a importância do fusível e do disjuntor para evitar o curto-circuito; v) aplicar adequadamente os conceitos físicos para compreender como ocorre o curto-circuito nas residências e como evitá-lo.

Como recursos didáticos, são utilizados notícias, reportagens, textos de livros didáticos, textos de sites, resolução de problemas, imagens, vídeos e simulação computacional.

Para a realização da $\mathrm{AD}$, serão necessários quatro aulas (com cinquenta minutos cada). A atividade está estruturada em sete etapas, e tem como ponto de partida a seguinte situação-problema: como ocorre o curto-circuito nas residências e como evitá-lo?

A primeira etapa inicia com a leitura de uma notícia sobre um incêndio, seguido pela análise inicial da referida situação-problema pelos estudantes. Na segunda etapa, serão discutidas as causas e consequências do curto-circuito, a partir da leitura, pelos estudantes, de um texto sobre o assunto, bem como pela representação de uma instalação elétrica em curto-circuito utilizando uma simulação computacional. A terceira etapa aborda a fiação elétrica, por meio da leitura de um texto sobre os cuidados com os fios nas instalações elétricas e da resolução de problemas. Na quarta etapa, os estudantes realizarão a leitura de um texto sobre fusível e disjuntor, seguido pela resolução de problemas. A quinta etapa consiste em os estudantes responderem novamente a situação-problema inicial, considerando o que foi trabalhado na atividade. Na sexta etapa, será realizada uma síntese do que foi estudado com o auxílio de vídeos. Para finalizar, na sétima etapa, os estudantes resolverão individualmente duas situações associadas ao conteúdo abordado nas etapas anteriores.

\subsection{AD 6 - Choque elétrico}

A AD 6 aborda o choque elétrico (caso 4), especificamente as causas e consequências (mini caso 4.1), a adequação das tomadas (mini caso 4.2) e a importância do aterramento (mini caso 4.3). Os temas de análise conceitual envolvidos são: corrente elétrica, lei de Ohm, potência elétrica, resistência elétrica e tensão elétrica.

Essa AD tem como objetivos específicos: i) relacionar adequadamente os conceitos de tensão, resistência e corrente elétrica; ii) identificar as causas e consequências do choque elétrico; iii) perceber a adequação das tomadas como um fator importante para evitar o choque elétrico; iv) analisar a importância do aterramento para evitar o choque elétrico; v) aplicar adequadamente os conceitos físicos para compreender os fatores que influenciam no choque elétrico e como evitá-lo.

Como recursos didáticos, são utilizados notícias, textos de livros didáticos, textos de sites, resolução de problemas, imagens, vídeos e simulação computacional.

Para a realização da $\mathrm{AD}$, serão necessários quatro aulas (com cinquenta minutos cada). A atividade está estruturada em seis etapas, e tem como ponto de partida a seguinte situação-problema: de quais fatores depende o choque elétrico e como evitá-lo?

A primeira etapa inicia com a leitura de uma notícia sobre choque elétrico, seguida pela análise inicial da referida situação-problema pelos estudantes. Na segunda etapa, os estudantes farão a leitura de dois textos sobre as causas e consequências do choque elétrico, além de resolver problemas sobre o assunto. A terceira etapa aborda o aterramento através da ilustração de um exemplo de aterramento, da leitura de dois textos sobre assunto, bem como da resolução de problemas. Na quarta etapa, será trabalhado a adequação das tomadas, por meio da resolução de problemas. Em seguida, na quinta etapa, será realizada uma síntese do que foi estudado na atividade por meio de um vídeo. Por fim, na sexta etapa, os estudantes resolverão individualmente duas situações associadas ao conteúdo abordado nas etapas anteriores.

\section{Considerações Finais}

Conforme explicitamos anteriormente, buscamos, neste trabalho, apresentar um conjunto de AD de Física mediadas por hipermídia, bem como discutir as potencialidades das mesmas para consubstanciar o desenvolvimento da FC no âmbito do ensino médio.

Tendo em vista um objetivo formativo mais amplo, o conjunto de AD construída possui objetivos gerais comuns, em consonância com os objetivos da TFC, a partir dos quais elas foram estruturadas. Sobre essa estrutura geral - situação-problema inicial, conteúdos de Física, resolução de problemas, análise da situação-problema, síntese, material extra e aplicação do conhecimento - ela foi comum a todas atividades e esteve em conformidade com elementos que a TFC apresenta para consubstanciar os aspectos necessários para o desenvolvimento da FC: caso, mini caso, tema de análise conceitual, comentário temático e travessia temática.

Anteriormente ao planejamento das AD, é importante explicitar que realizamos uma pesquisa bibliográfica em publicações de trinta e três periódicos das áreas de ensino e pesquisa em ensino de Física e Ciências, abrangendo o período de 1990 a 2017. O objetivo central foi, a partir da seleção e análise de artigos cuja ênfase estivesse na FC no referido âmbito, buscar subsídios para a elaboração e avaliação das AD de Física.

Nessa pesquisa, encontramos nas propostas analisadas uma diversidade de metodologias de ensino, instrumentos de avaliação e focos de avaliação. Isso contribuiu significativamente para a estruturação das AD de Física em 
consonância com os pressupostos teórico-metodológicos da TFC.

Em relação ao planejamento do conjunto de atividades, explicitamos a dificuldade que encontramos em estruturálas com base nesses pressupostos da TFC. Dentre as principais dificuldades, citamos: na escolha dos casos, definir o grau de complexidade adequado; na escolha dos mini casos, definir a quantidade adequada, de modo a considerarmos apenas aqueles que nos permitissem abordar aspectos que fossem de fato relevantes para cada caso; e na escolha dos temas de análise conceitual, definir a quantidade adequada de temas, optando apenas por aqueles que de fato fossem relevantes para a compreensão dos casos e mini casos. Essas dificuldades resultaram em diversas reformulações dos casos, mini casos e temas ao longo do processo de construção das AD.

A respeito da estruturação das $\mathrm{AD}$, encontramos dificuldade também em elaborar uma situação-problema inicial que abrangesse o(s) mini caso(s) e o caso a serem abordados. Ainda, na aplicação do conhecimento, o desafio foi elaborar situações novas que, de fato, envolvessem a recontextualização de conhecimentos; tendo clareza do quão diferente das situações anteriores precisa ser essa nova situação, de qual a "distância" adequada entre elas.

Essas dificuldades estão em conformidade com a afirmação de Marques, Carvalho e Guimarães [27], quando sublinham que a implementação da TFC é uma tarefa difícil, tendo em vista a diversidade de aspectos envolvidos. Nesse sentido, entendemos necessário explicitar as referidas dificuldades, de modo a apontar possíveis caminhos que seguimos no intuito de contorná-las, o que pode contribuir na elaboração de futuras ações nesse sentido.

Consideramos importante também mencionar a construção da Matriz Temática [23] para a sistematização dos casos, mini casos e temas de análise conceitual escolhidos. Em relação às contribuições da mesma para o planejamento das AD de Física, destacamos o fato de possibilitar: i) a visualização dos casos e mini casos que têm afinidades temáticas, ou seja, que são analisados pelos mesmos temas; ii) a visualização das possibilidades tanto de comentário temático quanto de travessia temática; iii) a concretização de um dos aspectos envolvidos nas múltiplas representações do conhecimento: o das múltiplas formas de organização do conhecimento, através de casos, mini casos e temas de análise conceitual interligados $[19,31,32]$; e iv) a consubstanciação de outros aspectos necessários para o desenvolvimento da FC, a saber: desconstrução de casos e mini casos por meio de diferentes temas; estabelecimento de ligações entre mini casos de diferentes casos; e promoção de diversas ligações entre conceitos e casos [20-23, 30].

Para além disso, cabe explicitar que, neste trabalho, a utilização das tecnologias educacionais em rede se deu a partir de uma perspectiva pedagógica mais ampla. Nesse sentido, as hipermídias foram utilizadas para a construção de uma estratégia de ensino que possuía um objetivo formativo mais abrangente - para além apenas do apren- dizado conceitual -, vinculado a uma compreensão de mundo articulada, na qual o desenvolvimento da FC é fundamental.

Em relação à hipermídia, essa se constitui no principal recurso didático para implementação da TFC [26, 38], contribuindo para promover características de FC [19]. Isso por que ela: i) possibilita que a informação possa ser interligada [38]; ii) permite que o mesmo material possa ser explorado de forma não linear, de acordo com diferentes percursos, de diferentes modos [19, 21, 25, 36]; iii) favorece o desenvolvimento, por parte dos estudantes, de suas próprias representações da informação ao construírem o conhecimento [30]; iv) possibilita apresentar um determinado conhecimento através de diferentes mídias, tais como textos, imagens, gráficos, animações e vídeos $[19,20]$. Desse modo, a hipermídia contribui para concretizar as múltiplas representações do conhecimento.

Nesse contexto, argumentamos que as AD de Física apresentadas e discutidas neste trabalho, pelos recursos didáticos utilizados e pela forma como estão estruturadas, apresentam potencialidades para consubstanciar os aspectos necessários para o desenvolvimento da FC em estudantes do ensino médio.

Uma das possibilidades de continuidade desse trabalho consiste na implementação do conjunto de $\mathrm{AD}$ de Física com estudantes do ensino médio, bem como avaliação dos resultados obtidos a partir de critérios coerentes com os aspectos necessários para o desenvolvimento da FC.

Na pesquisa bibliográfica referida, constatamos que a menor parte dos artigos encontrados esteve relacionada à elaboração, implementação e/ou avaliação de atividade. Nesse sentido, destacamos a pertinência e inovação de nosso trabalho que, através da elaboração de AD de Física mediadas por hipermídia, buscou apresentar e discutir uma ação concreta, em consonância com os pressupostos da TFC, voltada ao ensino de Física, o que se mostrou desafiador devido a pequena quantidade de trabalhos relacionados à temática, no âmbito do ensino de Física no Brasil.

\section{Referências}

[1] BRASIL, PCN+ Ensino Médio: Orientações educacionais complementares aos Parâmetros Curriculares Nacionais (Ministério da Educação, Brasília, 2002).

[2] BRASIL, Orientações Curriculares para o Ensino Médio (Ministério da Educação, Brasília, 2006).

[3] BRASIL, Diretrizes Curriculares Nacionais da Educação Básica (Ministério da Educação, Brasília, 2013).

[4] T. Pessoa e F. Nogueira, em Educação e contemporaneidade: pesquisas científicas e tecnológicas, editado por A. Nascimento e T. Hetkowski (EDUFBA, Salvador, 2009).

[5] J.I. Pozo e M.A.G. Crespo, A aprendizagem e o ensino de ciências: do conhecimento cotidiano ao conhecimento científico (Artmed, Porto Alegre, 2009).

[6] R.J. Spiro e J.C. Jehng, em Cognition, Education, and Multimedia: Exploring Ideas in High Technology, editado por D. Nix e R.J. Spiro (Lawrence Erlbaum Associates, Hillsdale, 1990). 
[7] A.A.A. Sousa, Aplicação da Teoria da Flexibilidade Cognitiva ao $1^{\circ}$ Ciclo do Ensino Básico: Um Estudo Sobre a Qualidade do Ambiente. Dissertação de Mestrado, Universidade do Minho, Braga (2004).

[8] A.A. Aleixo, FlexQuest no Ensino de Ciências: Incorporando a Teoria da Flexibilidade Cognitiva na WebQuest. Dissertação de Mestrado, Universidade Federal Rural de Pernambuco, Recife (2008).

[9] M.P.P. Echeverría e J.I. Pozo, em A solução de Problemas, editado por J.I. Pozo (Artmed, Porto Alegre, 1998).

[10] F. Rezende e C.S.D. Cola, Ensaio Pesquisa em Educação em Ciências 6, 1 (2004).

[11] D.I. Machado e R. Nardi, Revista Brasileira de Ensino de Física 28, 473 (2006).

[12] T. Da Silva, Caderno Brasileiro de Ensino de Física 26, 533 (2009).

[13] T. Da Silva, Caderno Brasileiro de Ensino de Física 29, 864 (2012).

[14] F. Rezende, J.J.F. Sousa e S.S. Barros, Caderno Brasileiro de Ensino de Física 29, 361 (2012).

[15] M. Grisolía e C.V. Grisolía, Revista Electrónica de Enseñanza de las Ciencias 8, 440 (2009).

[16] C.P. Coutinho, em Anais da $4^{\underline{a}}$ Conferencia IberoAmericana en Sistemas, Cibernética e Informática (Orlando, 2005).

[17] L.A.Z. Salgado, em $31^{\circ}$ Congresso Brasileiro de Ciências da Comunicação (Natal, 2008).

[18] C.G. Guerra, em $3^{\circ}$ Seminário de IESDT (Portalegre, 2012).

[19] R.J. Spiro, P.J. Feltovich, M.J. Jacobson e R.L. Coulson, em Constructivism and the Technology of Instruction: A Conversation (Lawrence Erlbaum Associates, Hillsdale, 1992).

[20] L.F.M.G. Pedro, A construção de materiais didácticos por recurso a um hipertexto de flexibilidade cognitiva: um estudo comparativo. Tese de Doutorado, Universidade de Aveiro, Aveiro, (2005).

[21] A.A.A. Carvalho, Os documentos hipermédia estruturados segundo a teoria da flexibilidade cognitiva: Importância dos comentários temáticos e das travessias temáticas na transferência do conhecimento para novas situaçôes. Tese de Doutorado, Universidade do Minho, Braga (1998).

[22] R.J. Spiro, R.L. Coulson, P.J. Feltovich e D.K. Anderson, em Proceedings of the 10th Annual Conference of the Cognitive Science Society (Hillsdale, 1988).

[23] A.A.A. Carvalho, Revista Portuguesa de Educação 13, 169 (2000).

[24] S.P.S.R. Pinho, Padrões de Interaç̧ão num Ambiente de Aprendizagem Colaborativa a Distância: Um estudo de caso com conteúdos estruturados segundo a Teoria da Flexibilidade Cognitiva. Dissertação de Mestrado, Universidade de Aveiro, Aveiro (2006).

[25] P. Dias, Revista Portuguesa de Educação 13, 141 (2000).

[26] E. Bolacha e F. Amador, Investigações em Ensino de Ciências 8, 31 (2003).

[27] C.G.C. Marques, A.A.A. Carvalho e N. Guimarães, Revista Portuguesa de Educação 17, 151 (2004).

[28] A.A.A. Carvalho, em Tecnologias na Educação: uma abordagem crítica para uma atuação prática, editado por M.B.C. Leão (UFRPE, Recife, 2011).
[29] A.A.A. Carvalho, C.S. Pinto e P.J.M. Monteiro, em $4^{\circ}$ Simpósio Internacional De Informática Educativa (Vigo, 2002).

[30] F. Rezende, M.A.C. Garcia e C.S.D. Cola, Investigações em Ensino de Ciências 11, 239 (2006).

[31] F. Rezende, Caderno Brasileiro de Ensino de Física 18, 197 (2001).

[32] R.V. Souza e M.B.C. Leão, Ciência \& Educação 21, 1049 (2015).

[33] A.A.A. Carvalho, C.S. Pinto, A.A. Baptista e P.J.M. Monteiro, em $1^{a}$ Conferencia Ibero-Americana en Sistemas, Cibernética e Informática (Orlando, 2002).

[34] A. Moreira, L. Pedro e F. Nogueira, em Tecnologias na Educação: Uma abordagem crítica para uma atuação prática, editado por M.B.C. Leão (UFRPE, Recife, 2011).

[35] F.C.G.C. Vasconcelos e M.B.C. Leão, Investigações em Ensino de Ciências 17, 37 (2012).

[36] R.J. Spiro, B.P. Collins e A.R. Ramchandran, em Video research in the Learning Sciences, editado por R. Goldman, R. Pea, B. Barron e S.J. Derry (Lawrence Erlbaum Associates, Mahwah, 2007).

[37] R.J. Spiro, B.P. Collins e A.R. Ramchandran, em Flexible learning in an information society, editado por B. Khan (Information Science Publishing, Hershey, 2007).

[38] M.B.C. Leão, F.N. Souza e A. Moreira, Indagatio Didactica 3, 108 (2011).

[39] D.N. Heidrich, Construção e avaliação de hipermídia educacional sobre tópicos de carboidratos. Tese de Doutorado, Universidade Federal de Santa Catarina, Florianópolis (2009). 